\title{
ÉDUCATION SANITAIRE POUR LA PRÉVENTION DES MALADIES: REVUE DE LA LITTÉRATURE
}

\section{RÉVISER L'ARTICLE}

GITIRANA, José Valdeci Almeida ${ }^{1}$, FONSECA, Rosa Maria Batista Pinheiro da², PILONETO, Fábio Marmentini ${ }^{3}$, BEVILAQUA, Luis Felipe Gaia ${ }^{4}$, ASSIS, Ingrid de ${ }^{5}$, CARDOSO, Ronald de Oliveira ${ }^{6}$

GITIRANA, José Valdeci Almeida. Et al. Éducation sanitaire pour la prévention des maladies: revue de la littérature. Revista Científica Multidisciplinar Núcleo do Conhecimento. Année. 06, Ed. 11, vol. 08, p. 134 à 147. Novembre 2021. ISSN: 2448-0959, Lien d'accès:

https://www.nucleodoconhecimento.com.br/sante/education-sanitaire, DOI: 10.32749/nucleodoconhecimento.com.br/sante/education-sanitaire

\section{RÉSUMÉ}

Actuellement, les politiques publiques, les actions gouvernementales et les différents programmes impliquant l'éducation sanitaire (ES) sont mis en œuvre en faveur de la santé de la population. En ce qui concerne le thème de la $E S$, il s'agit d'un thème à multiples facettes, convergeant vers diverses conceptions personnelles, que ce soit dans le domaine de l'éducation ou de la santé, qui peuvent entraîner des compréhensions divergentes. En raison de la complexité du thème, le problème survient en raison du manque de compréhension de ce qui est réellement la ES face à la population, ce qui dissout l'importance de la ES pour les mesures préventives contre différentes maladies, ce qui génère un coût budgétaire élevé en santé publique. Dans ce contexte, cet article a pour question principale : comment

\footnotetext{
${ }^{1}$ Diplômé en pharmacie, diplômé en analyse clinique, actuellement universitaire en médecine.

${ }^{2}$ Médecin spécialiste.

${ }^{3}$ Diplômé en administration des affaires. Diplômé en commerce extérieur, actuellement étudiant en médecine.

${ }^{4}$ Diplômé en génie alimentaire, diplômé en génie des procédés industriels, CBA en gestion des affaires et actuellement étudiant en médecine.

${ }^{5}$ Médecin spécialiste.

${ }^{6}$ Médecin spécialiste.
}

RC: 102788

Disponible en: https://www.nucleodoconhecimento.com.br/sante/education-sanitaire 
l'éducation sanitaire peut-elle contribuer à la prévention des maladies dans la population ? L'étude visait à présenter l'ES pour la prévention des maladies (PD), en abordant ses concepts, ainsi qu'en soulignant les bases juridiques qui assurent l'ES à la population. À cette fin, l'étude a été menée par le biais d'une revue de la littérature par le site de recherche « Google Scholar », «Scielo » et «PubMed ». Par le biais des descripteurs : Éducation sanitaire; L'éducation sanitaire en tant que prévention des maladies; éducation sanitaire pour la population, où des études ont été choisies qui présentaient les ES axées sur la PD. II est conclu que les ES sont l'éducation à la connaissance de soi et à la réflexion sur leur propre santé, ainsi que la pleine conscience que quelque chose ne va pas, attribuant la réflexion critique du sujet concernant leurs habitudes et leurs mesures préventives. II est appliqué à travers le programme scolaire de l'école primaire ainsi que par les programmes gouvernementaux dans les centres de santé, afin d'éduquer la société à sensibiliser aux mesures préventives et à promouvoir une meilleure qualité de vie, empêchant ainsi la propagation des maladies.

Mots-clés: Éducation, Prévention des maladies, Promotion de la santé, Population.

\section{INTRODUCTION}

L'éducation à la santé (ES) est une pratique sociale dont le processus d'apprentissage contribue à la formation d'une conscience personnelle critique concernant les problèmes de santé eux-mêmes, en faisant réfléchir le citoyen sur sa réalité, et à partir de là, à rechercher des solutions et une organisation en faveur de sa santé et de sa santé collective, aboutir à une promotion de la santé (PS) (BISCARDE; PEREIRA-SANTOS( SILVA, 2014).

Les origines et les conceptions du PS ont commencé avec l'avènement de la SSc au début du 20ème siècle, à travers l'observation de la diminution des taux de maladie résultant des pratiques éducatives effectuées par les "hygiénistes » de [7] l'époque (ENRIA; STAFOLANI, 2010). À cette époque, la signification de PS était attribuée aux actions d'ES, dans le but d'une meilleure qualité de vie. Cependant, le SSc a actuellement une caractéristique amplifiée, car il est considéré comme l'un des 
principaux dispositifs pour la viabilité de la PS, conduisant au développement de la responsabilité individuelle et de la prévention des maladies (PD) (LOPES; Grêle; XIMENES, 2010; FALKENBERG et al., 2014; SPERANDIO; SPERANDIO, 2018).

Bien que l'ES soit comprise comme l'une des mesures pour le (JANINI; BESSLER; VARGAS, 2015; SPERANDIO; SPERANDIO, 2018), c'est un thème aux multiples facettes, convergeant vers diverses conceptions personnelles, que ce soit pour les personnes dans le domaine de l'éducation, ou dans le domaine de la santé, ce qui peut entraîner des compréhensions divergentes. (SCHALL; STRUCHINER, 1999).

En raison de la complexité du thème, le problème se pose en raison du manque de compréhension de ce qui est réellement la SSc face à la population, dissolvant l'importance de la SSc pour les mesures préventives contre différentes maladies. Pour cette raison, de nombreuses maladies qui pourraient être évitées sont souvent considérées comme ayant un impact majeur sur le budget public. Par conséquent, la présente étude devait répondre à la question: Comment l'éducation à la santé peutelle contribuer à la prévention des maladies dans la population? Par le biais d'une revue de la littérature, l'objectif général a cherché à présenter comment les SS peuvent promouvoir la DP, en abordant ses concepts, ainsi qu'en soulignant les bases juridiques qui assurent la SSc à la population.

À cette fin, l'étude a été menée par le biais d'une revue de la littérature par le site de recherche "Google Scholar», "Scielo» et «PubMed». Par le biais des descripteurs : Éducation à la santé; L'éducation à la santé en tant que prévention des maladies; Éducation à la santé pour la population. En guise d'inclusion, les articles et les documents ont été choisis entre 2018 et 2020, composant la structure nécessaire pour atteindre l'objectif souhaité. Par la suite, 20 articles ont été trouvés, dont 8 ont été sélectionnés. Les articles qui répondaient à l'objectif et à la question du guide et qui se trouvaient dans le délai proposé ont été inclus. Les études destinées uniquement aux professionnels de la santé ou aux étudiants de premier cycle qualifiés dans le domaine de la santé ont été exclues. 
Le thème est d'actualité de nos jours, car l'ES peut influencer la capacité de la population à réfléchir sur la lutte contre différentes maladies, entraînant de meilleures habitudes de vie. II peut être pertinent pour les profanes ou pour les étudiants et les professionnels du domaine académique, servant de source pour les études futures car il éclaire le concept d'ES pour la population comme indissociable du PD, offrant un langage facile à comprendre.

\section{L'INDISSOCIABILITÉ DE L'ÉDUCATION SANITAIRE ET DE LA PRÉVENTION DES MALADIES}

L'ES est planifié et appliqué de différentes manières, une telle modalité d'enseignement peut être destinée aux étudiants de premier cycle dans le domaine de la santé; pour les professionnels de santé ; pour l'enseignement scolaire et pour la population en général. En effet, il tend à être compris de différentes manières, répondant à la formation culturelle et sociale de chacun. Cependant, cette étude met l'accent sur l'ES pour la population comme indissociable du PD, puisque l'ES est « [...] lié aux objectifs généraux de santé publique : prévention des maladies, protection et promotion de la santé de l'individu, de la famille et de la communauté ». (PIMONT, 1997, p. 17).

Pour mieux contextualiser la compréhension de cette inséparabilité, il est important de souligner que les ES en sont un :

\footnotetext{
Processo educativo de construção de conhecimentos em saúde que visa à apropriação temática pela população [...]. Conjunto de práticas do setor que contribui para aumentar a autonomia das pessoas no seu cuidado e no debate com os profissionais [...] a fim de alcançar uma atenção de saúde de acordo com suas necessidades. (BRASIL, 2012, p. 19).
}

Comme présenté dans le " Glossaire thématique: gestion du travail et éducation à la santé », l'ES provoque l'autonomie souhaitable afin que les personnes puissent s'exprimer efficacement afin de prouver leurs besoins spécifiques dans leurs soins cliniques, contribuant ainsi aux soins du professionnel de la santé pour traiter rapidement les maladies, ainsi que prévenir d'autres maladies secondaires. 
Complétant cette définition, il est présenté dans le document de la Fondation nationale de la santé (FUNASA) Brasil (2007), que l'ES est une pratique sociale, réaffirmant que son intention se transforme en une transformation pour tout type de personne «[...] rompant avec le paradigme de la conception statique de l'éducation comme transfert de connaissances, de compétences et de compétences ». (BRASIL, 2007, p. 20).

Pimont (1997, p. 15) résumé et analyse différents concepts adoptés, en soulignant que l'ES vise à fournir :

1) A consciência do direito à saúde, dentro do grau de desenvolvimento do grupo social no qual vive o indivíduo; 2) 0 conhecimento sobre saúde; 3) O conhecimento dos serviços para a saúde que a comunidade possua e sua consequente utilização; 4) O conhecimento e a utilização das práticas de saúde.

Cependant, l'auteur assimile que pour que cet enseignement soit transformé en connaissance afin de promouvoir PS et la PD, il est nécessaire qu'une planification soit structurée, atténuant une méthodologie éducative adéquate, composée d'instruments auxiliaires, ce qui peut alors aboutir à un processus éducatif constant.

Selon Altuve (1996), pour que l'ES devienne une connaissance, l'organisation de la communauté devient déjà une méthode qui produit un changement social, car au milieu des efforts et des techniques, son application entraîne l'induction et la promotion de changements sociaux significatifs, englobant tous les secteurs sociaux, tels que la relation entre les niveaux économique, politique et éducatif.

Par conséquent, selon les auteurs cités, la ES peut être inséparable de la PD à la population, lorsqu'elle est transmise méthodologiquement. Cependant, la présente étude n'entend pas approfondir ces méthodologies, mais conceptualise plutôt l'ES comme un PD pour la population. 


\subsection{BASES JURIDIQUES DE L’ÉDUCATION SANITAIRE DE LA POPULATION}

En mettant l'accent sur l'ES au Brésil, en août 1971, la loi 5692 a été créée, reformulant le programme scolaire dans l'enseignement primaire et secondaire, mettant en œuvre un enseignement axé sur le domaine de la santé tel que présenté à l'article 7 :

Art. $7^{0}$ Será obrigatória a inclusão de Educação Moral e Cívica, Educação Física, Educação Artística e Programas de Saúde nos currículos plenos dos estabelecimentos de ำ e $2^{\circ}$ graus, observado quanto à primeira o disposto no Decreto-Lei n. 369, de 12 de setembro de 1969. (Vide Decreto $n^{\circ} 69.450$, de 1971)

Par la suite, des programmes tels que "Saúde na escola" ont été utilisés comme une stratégie intersectorielle développée dans le milieu scolaire et ses environs, articulant des actions visant la PS et la qualité de vie, ainsi qu'une réflexion sur le PD et la connaissance du Système de santé unifié (SUS) (BRASIL, 2012). Ce contexte peut être mis en évidence par le fait que des actions pour l'ES sont mises en œuvre tout au long de la vie, dès le premier cycle de l'enseignement scolaire, afin que les personnes développent progressivement un sens critique, réflexif et conscient.

En faisant éclater positivement la portée des programmes visant les ES et les PD, différentes lois ont été créées, selon les bases juridiques présentées dans le tableau 1 ci-dessous: 
Tableau 1 - Bases juridiques de l'éducation à la santé.

\begin{abstract}
A Lei n. ${ }^{\circ}$ 8.080/1990, que regulamenta o SUS, em seu Capítulo I, Art. $5^{\circ}$, Item III, define como um dos objetivos e atribuições deste Sistema: "a assistência às pessoas por intermédio de ações de promoção, proteção e recuperação da saúde, com a realização integrada das ações assistenciais e das atividades preventivas". A mesma Lei, em seu Capítulo II, Artigo 7_ ${ }^{\circ}$, Item VIII, estabelece a
\end{abstract} "participação da comunidade."

A Lei $\mathbf{N}^{\circ} \mathbf{8 . 1 4 2}$, de 28 de dezembro de 1990, define a participação da comunidade na gestão SUS e no seu artigo $1^{\circ}$ "as instâncias colegiadas de controle social: I) a Conferência de Saúde, e II) o Conselho de Saúde."

A Norma Operacional Básica - SUS 1/1996 (NOB/96), que redefine as responsabilidades dos estados, do Distrito Federal e da União e consolida as responsabilidades dos municípios, cita como um dos papéis do gestor federal e do estadual a "Educação em Saúde

A Educação em Saúde na Funasa está ancorada em seu Regimento Interno, Portaria GM n ${ }^{\circ} 1.776$ de 8/9/2003, assinada pelo Ministro da Saúde que estabelece as competências da Assessoria de Comunicação e Educação em Saúde-Ascom e seus setores, dentro das competências dos demais setores do órgão. (...)

Lei n. 9.394, de 20 de dezembro de 1996 (1996). Estabelece as diretrizes e bases da educação nacional.

DCN - EF 1988 - Estabelecer as Diretrizes Curriculares Nacionais para o Ensino Fundamental: Saúde articulada à educação e à vida cidadã.

PCN - CN 1988 - Estabelecer as Referências Nacionais Comuns para o Ensino das Ciências Naturais: Saúde como estado de equilíbrio dinâmico do corpo.

PCN - TT Saúde 1988 - Apresentar a saúde como um Tema Transversal: Educação para a saúde

PCN - EM 1999 - Apresentar os Parâmetros Curriculares Nacionais para o Ensino Médio: Degradação ambiental e agravos à saúde humana.

PCN + 2002 - Apresentar Orientações Curriculares complementares aos PCN: Saúde e as condições de vida das pessoas.

OCEM 2006 - Apresentar sugestões para as práticas pedagógicas e organização dos

currículos do Ensino Médio: Ambiente e saúde.

DCNEB 2013 - Estabelecer Orientações Nacionais para a Educação Básica. Saúde como campo de conhecimento e de serviços.

BNCC - $\mathbf{1}^{\mathbf{a}}$ versão 2015 - Estabelecer uma Base Nacional Comum para os currículos da Educação Básica, com versão inicial disponibilizada para consulta pública, estabelecendo cuidado com a saúde da população.

BNCC - $2^{\mathbf{a}}$ versão 2016 - Estabelecer uma Base Nacional Comum para os currículos da Educação Básica, com versão elaborada após contribuições da sociedade, para o ambiente e saúde.

BNCC $3^{a}$ versão 2016 - Estabelecer uma Base Nacional Comum para os currículos da Educação Básica com versão disponibilizada após contribuições institucionais, para promoção dos cuidados com a saúde.

BNCC versão final (EI e EF) 2016 - Estabelecer uma Base Nacional Comum para os currículos da Educação Infantil e do Ensino Fundamental, compondo a versão final, com o objetivo de promover os cuidados com a saúde.

BNCC EM 2018 - Estabelecer uma Base Nacional Comum para os currículos do Ensino Médio, promoção do cuidado com a saúde.

Source : Adapté du Brésil (2012) ; Sousa, Guimarães et Amoureux (2019). 
Le tableau présente les lois, où certaines bases éducatives pour l'applicabilité de ES dans les écoles sont mises en évidence, même si ces directives visent l'environnement scolaire, les objectifs du programme guident la formation des personnes qui comprennent l'importance de ES et qui grâce à cette connaissance, ils peuvent promouvoir les soins de santé personnelle et de la population, composant également l'amélioration du PD.

Aujourd'hui, les Nations Unies présentent le troisième objectif de "l'Agenda 2030 " et, avec le soutien mondial, il est prévu à travers l'action " d'assurer une vie saine et de promouvoir le bien-être de tous et de tous, à tous les âges »(ODS, 2021), indépendamment de la classe sociale, de la race ou de la couleur.

La place à l'ordre du jour 2030 est conforme à la perspective de SHALL (1999), où elle atténue que la définition des ES doit être plus large qu'une simple action résultant de l'PS, car elle est axée sur la population passe par un processus global de participation active de l'ensemble de la population dans le contexte de sa vie quotidienne et pas seulement des personnes qui risquent de tomber malades, être alors un moyen de prévenir les maladies pour les personnes en bonne santé, d'établir une meilleure qualité de vie et un plus grand bien-être pour tous.

\section{2 ÉTUDES LIÉES À LA PRÉVENTION DES MALADIES PAR L'ÉDUCATION À LA SANTÉ}

Ensuite, les principales conclusions sur la PD seront présentées à la suite de l'application de la ES, disponible dans la littérature scientifique, entre les années 2018 et 2020. Ensuite, le tableau 2 présente une synthèse des 8 articles précédemment sélectionnés présentés dans l'ordre chronologique, où les méthodologies utilisées dans chaque étude seront expliquées plus loin. 
Tableau 2 - Études liées à l'éducation à la santé en tant que prévention des maladies.

\begin{tabular}{|c|c|c|}
\hline Auteur & But & Conclusion \\
\hline $\begin{array}{l}\text { Dias et al. } \\
(2018)\end{array}$ & $\begin{array}{l}\text { Rapportez l'expérience d'une } \\
\text { action d'éducation à la santé } \\
\text { avec des élèves d'une école } \\
\text { publique dans les zones rurales. }\end{array}$ & $\begin{array}{l}\text { Les enfants sont exposés au } \\
\text { risque de contamination par des } \\
\text { parasites, compte tenu des } \\
\text { conditions sanitaires qu'ils } \\
\text { habitent, ce qui témoigne de la } \\
\text { nécessité d'étendre les actions } \\
\text { d'éducation sanitaire sur } \\
\text { l'importance de la prévention des } \\
\text { parasites. }\end{array}$ \\
\hline $\begin{array}{l}\text { Salci, } \\
\text { Meirelles e } \\
\text { Silva (2018) }\end{array}$ & $\begin{array}{l}\text { Comprendre comment les } \\
\text { membres de la Stratégie de } \\
\text { santé familiale s'organisent pour } \\
\text { l'éducation à la santé, dans la } \\
\text { gestion et la prévention des } \\
\text { complications chroniques du } \\
\text { diabète sucré dans les soins de } \\
\text { santé primaires }\end{array}$ & $\begin{array}{l}\text { On considère que la pratique de } \\
\text { l'éducation à la santé pour les } \\
\text { personnes atteintes de diabète } \\
\text { mellitus in Primary Health Care } \\
\text { compose un contexte à multiples } \\
\text { facettes, avec de multiples } \\
\text { obstacles à son exécution. }\end{array}$ \\
\hline $\begin{array}{l}\text { Gomes et } \\
\text { al. (2019) }\end{array}$ & $\begin{array}{l}\text { Signaler l'action éducative dans } \\
\text { un groupe d'hommes afin de } \\
\text { rechercher une meilleure } \\
\text { compréhension du cancer du } \\
\text { pénis et des mesures de } \\
\text { prévention et de détection } \\
\text { précoce. }\end{array}$ & $\begin{array}{l}\text { Les connaissances sur le cancer } \\
\text { du pénis doivent encore être } \\
\text { mieux élaborées chez les } \\
\text { hommes. }\end{array}$ \\
\hline $\begin{array}{l}\text { Figueiredo; } \\
\text { Antunes e } \\
\text { De Miranda }\end{array}$ & $\begin{array}{l}\text { Mener à la création de politiques } \\
\text { d'éducation en matière de santé } \\
\text { publique, avec des soins }\end{array}$ & $\begin{array}{l}\text { Les politiques publiques sont } \\
\text { essentielles pour la } \\
\text { communication et le renforcement }\end{array}$ \\
\hline
\end{tabular}


scolaires spécifiques et de toutes les mesures et

l'aiguillage des élèves obèses directives dans les domaines de vers les bureaux de santé l'éducation et de la santé. L'interdisciplinarité favorisera les transformations souhaitées, à travers des actions de prévention, dans le but de réduire l'implication de l'obésité et de ses conséquences, dans les groupes d'âge plus jeunes, ainsi que la réduction des maladies cardiovasculaires dans la phase précoce de la vie et à l'âge adulte.

Ricci et al. Rapportez (2019) l'expérience

d'universitaires de l'Université fédérale du Mato Grosso do Sul (UFMS) lors d'une action développée avec le groupe de femmes enceintes d'une unité de santé de base à Campo Grande - MS (UBS).
La réalisation de l'éducation sanitaire sur les infections sexuellement transmissibles (IST) pendant la grossesse s'est avérée efficace pour assurer le dialogue, l'interaction, l'échange d'expériences, de connaissances et d'expériences, et la construction conjointe de connaissances, étant alors démontrées comme importantes pour la promotion de la santé et la prévention des maladies, et pour le développement d'une sensibilisation critique et réfléchie des femmes enceintes à leur processus de santé-maladie, en les rendant actifs dans le 


\begin{tabular}{|c|c|c|}
\hline & & processus d'apprentissage. \\
\hline $\begin{array}{l}\text { De Sousa } \\
\text { Gonçalves } \\
\text { et al. (2020) }\end{array}$ & $\begin{array}{l}\text { Connaître le travail d'éducation } \\
\text { sanitaire d'un service de santé } \\
\text { de base dans la municipalité } \\
\text { d'Imperatriz - MA. }\end{array}$ & $\begin{array}{l}\text { Il est perçu qu'il est essentiel } \\
\text { d'améliorer constamment les } \\
\text { connaissances et la pratique des } \\
\text { professionnels pour développer } \\
\text { des actions d'éducation à la } \\
\text { santé. II est nécessaire d'élargir } \\
\text { les actions éducatives dans le } \\
\text { domaine de responsabilité de } \\
\text { chaque équipe, ainsi qu'une } \\
\text { participation plus active de tous } \\
\text { les membres de l'équipe de } \\
\text { stratégie familiale, ainsi que des } \\
\text { professionnels du centre de } \\
\text { soutien à la santé familiale } \\
\text { (NASF). }\end{array}$ \\
\hline $\begin{array}{l}\text { Lima et al. } \\
(2020)\end{array}$ & $\begin{array}{l}\text { Décrivez l'expérience en tant } \\
\text { qu'étudiants en soins infirmiers } \\
\text { dans des activités d'éducation à } \\
\text { la santé pour la prévention des } \\
\text { maladies cardiovasculaires chez } \\
\text { les personnes âgées. }\end{array}$ & $\begin{array}{l}\text { Les pratiques d'éducation à la } \\
\text { santé sont transformatrices et } \\
\text { apportent d'importantes } \\
\text { contributions aux soins infirmiers } \\
\text { gérontologiques, car elles } \\
\text { permettent l'exercice d'une } \\
\text { pensée critique et contribuent à la } \\
\text { formation de professionnels } \\
\text { engagés à travailler dans un } \\
\text { modèle de soins, basé sur des } \\
\text { preuves scientifiques et capable } \\
\text { de répondre aux besoins de la } \\
\text { population âgée, qui a augmenté } \\
\text { au fil des ans. }\end{array}$ \\
\hline Costa et al. & ignaler une action d'éd & eloppée a servi \\
\hline
\end{tabular}




\begin{tabular}{|l|l|l} 
à la santé a eu lieu dans le & mécanisme de consolidation des \\
cadre des soins de santé & aspects qui constituent \\
primaires, dont la proposition & l'éducation à la santé, réitérant le \\
était de prévenir le cancer du & rôle fondamental des soins \\
sein de la reconnaissance & infirmiers dans le processus de \\
préalable de ses manifestations, & soins. \\
ainsi que le maintien d'habitudes & \\
saines, visant à promouvoir la & \\
santé de ces femmes.
\end{tabular}

Source : L'auteur.

Dias et al. (2018), à travers un rapport d'expérience avec une méthode d'activité extra-classe de la composante curriculaire «Projet d'intégrateur scientifique des soins infirmiers de premier cycle » de Faculdade Verde Norte, tenu en octobre 2016 à l'école municipale Odilon Antunes, dans le village de Poções de la ville de Monte Azul, une action d'éducation à la santé avec la participation de 30 étudiants, 03 enseignants et 09 parents. L'action s'est produite dans les étapes d'exposition dialogique du contenu et dans l'affichage du déclencheur de discussion vidéo, étant débattue dans la roue des conversations avec le public. Ainsi, l'étude a fourni des mesures préventives pour la contamination par des maladies parasitaires.

Salci; Meirelles e Silva (2018) a mené une étude qualitative, composant la pensée complexe comme référence théorique et la recherche évaluative comme référence méthodologique. Pour la collecte de données, 38 professionnels de la santé ont interrogé des patients atteints de diabète sucré. Les agents de santé ont observé et analysé les dossiers médicaux des patients, grâce à la triangulation des données avec le support du logiciel ATLAS.ti. Les auteurs ont observé que, selon la complexité du thème à multiples facettes, la SSc peut promouvoir la DP primaire et secondaire.

Corroborant cette complexité, Figueiredo; Antunes et De Miranda (2019) ont mené une recherche de revue bibliographique, en appliquant la méthode de recherche 
exploratoire. Les auteurs ont lié la prévention des comorbidités et des maladies cardiovasculaires aux politiques publiques de la SSc en milieu scolaire. Par conséquent, ils ont souligné que l'interdisciplinarité qui implique la SSc peut promouvoir les transformations souhaitées chez les étudiants, à travers des actions de prévention, la prévention des maladies cardiovasculaires dans l'enfance et l'âge adulte.

Lima et al. (2020) dans une étude similaire, mais destinée au public âgé, ils ont observé à travers un rapport d'expérience, à travers la méthode descriptive, avec une approche qualitative, expérimentée dans le « Programme de soins de santé pour les personnes âgées - PROASI », que les activités de SSc servent d'outil dans la prévention des maladies cardiovasculaires, provoquant une pensée critique-réflexive à leur propre santé et qualité de vie.

Gomes et al. (2019) à travers l'étude descriptive exploratoire du type de rapport d'expérience, ils ont appliqué une activité réalisée en mars 2018 dans une église située à la périphérie de Belém/Pará. Le public participant était composé de vingttrois hommes âgés de 18 à 50 ans qui ont été soumis à des conférences avec la présentation de bannières; explication du cancer du pénis; soins de la PD et démonstration de prothèses péniennes. Les auteurs ont observé à travers la roue de conversation avec les participants, que peu connaissaient sur le thème du cancer du pénis. Par conséquent, les auteurs ont mis en garde contre l'importance de mettre en œuvre des programmes d'ES axés sur la PD tels que le cancer du pénis, car avec des programmes axés sur la ES, cette maladie et d'autres peuvent être considérablement réduites dans la population.

Toujours dans le contexte de la prévention du cancer, Costa et al. (2020), a mené une étude descriptive avec une approche qualitative, du type de rapport d'expérience. L'étude a été réalisée dans le cadre de l'hôpital, trouvé dans la région périphérique de la ville de Belém-PA, dans une unité de santé avec des professionnels qui promeuvent une modalité de traitement de santé axée sur le niveau primaire des maladies. Les traitements destinés au public féminin sont consolidés dans la mise en œuvre du "Programa Saúde da Mulher », dans lequel 
les patientes sont traitées avec des soins prénatals; accouchement; post-partum; prendre soin des familles; programmes de prévention du cancer du sein et de l'utérus; IST. Ce programme est composé de professionnels de divers domaines de la santé, ainsi que des différents types d'analyses cliniques pour l'adoption de mesures préventives, favorisant le diagnostic préalable de pathologies telles que le cancer. Avec la mise en œuvre d'une action éducative dans le programme, les professionnels de la santé ont suivi les patients dans leurs consultations, et de la même manière que l'étude de Gomes et al. (2019) dossiers exposés; présentations anatomiques du sein; entre autres activités éducatives et interactives. Par conséquent, les auteurs ont élucidé que les 16 participantes avaient une plus grande réflexion sur l'importance des soins personnels et comprenaient l'importance de la ES pour la prévention du cancer du sein.

Au regard de cette réflexion évoquée par Gomes et al. (2019), Ricci et al. (2019) corroborent que ce processus de prise de conscience réflexive passe par le dialogue ; interaction; échange d'expériences; connaissances et expériences et la construction conjointe de connaissances, ces éléments étant mis en évidence comme importants pour le PS et le PD. Cette observation a été mise en évidence dans l'étude qui a eu lieu le 15 mars 2017, dans l'après-midi dans la salle de réunion d'une unité de santé de base à Campo Grande - MS. Une méthodologie a été utilisée de manière stratégique composée de pratiques ludiques dans le but d'interactivité des participants afin qu'à travers la pratique pédagogique, ils puissent acquérir une compréhension du sujet en question. Initialement, les participantes enceintes étaient invitées par le biais d'appels d'agents communautaires de la région du bureau de santé. Ensuite, l'action a commencé avec la technique brise-glace « Passer le bébé ", dans laquelle les participants ont été présentés en s'identifiant avec leur nom, leur âge gestationnel et le choix du nom de l'enfant. Grâce à la dynamique, les femmes enceintes ont pris conscience des maladies qui pourraient être évitées pendant la grossesse, ainsi que de la PD chez le bébé lui-même.

Pour que cette conscience réflexive implique la famille, De Sousa Gonçalves et al. (2020), a réalisé un travail quantitatif avec une approche analytique, basé sur une 
collection rétrospective des actions de ES menées de février à septembre 2019. La recherche a été réalisée dans une unité de santé de base (UBS) de la municipalité d'Imperatriz dans l'État du Maranhão, dans laquelle elle était composée de 3 équipes de stratégie de santé familiale. Les données ont été collectées à partir de la collecte de documents avec des enregistrements des actions des ES effectuées dans I'UBS et dans les écoles de la région, couvertes par chaque équipe au cours de la période susmentionnée, mis à disposition par la direction de l'unité de santé. Les auteurs ont analysé que grâce à la ES, non seulement les maladies chroniques, sexuelles ou infectieuses peuvent être évitées, mais aussi les mesures prophylactiques peuvent résulter de programmes qui aident ces familles.

\section{CONSIDÉRATIONS FINALES}

ES peut promouvoir la PD par le biais de programmes de politique publique visant à mettre en œuvre des programmes éducatifs ES dans les écoles, UBS, les réseaux hospitaliers ainsi que les postes de santé. Pour cela, ses concepts peuvent être largement présentés, car l'ES est un thème complexe et multiforme.

À travers les résultats, il est considéré que l'ES peut stimuler le PD avec le PS pour la population, lorsqu'il y a un engagement de ce public cible à travers des activités méthodologiquement planifiées et appliquées avec la contribution d'instruments spécifiques pour le développement de l'apprentissage. En ce qui concerne la PD, il existe plusieurs prophylaxie trouvée dans les études, à savoir : cardiovasculaire, diabète, maladies chroniques non transmissibles, maladies infectieuses sexuellement transmissibles, maladies parasitaires, cancers, entre autres existantes.

Dans le contexte des lois et des bases juridiques de la ES, il vise à une grande attente chez les étudiants des premiers stades et du lycée, car le processus éducatif n'est pas imposé à l'âge adulte mais peut être construit au cours de la vie, devenant connaissance.

Par conséquent, en réponse à la question directrice, il est conclu que l'ES est une éducation à la connaissance de soi et à la réflexion sur sa propre santé, ainsi que la 
pleine conscience que quelque chose ne va pas, attribuant la réflexion critique du sujet sur ses habitudes et mesures préventives. , prévenant ainsi la maladie. II est appliqué à travers le programme scolaire de l'éducation de base ainsi que par les programmes gouvernementaux dans les centres de santé, afin d'éduquer la société à sensibiliser aux mesures préventives et à la promotion d'une meilleure qualité de vie.

\section{RÉFÉRENCES}

ABREU JUNIOR, Laerthe de Moraes; CARVALHO, Eliane Vianey de. O discurso médico-higienista no Brasil do início do século XX. Trabalho, Educação e Saúde, v. 10, p. 427-451, 2012.

ALTUVE. R. L. O. La organización de la comunidad como un proceso educativo. Cuadernos de la Escuela de Salud Pública (Caracas) 4:9-28, 1966

BISCARDE, Daniela Gomes dos Santos; PEREIRA-SANTOS, Marcos; SILVA, Lília Bittencourt. Formação em saúde, extensão universitária e Sistema Único de Saúde (SUS): conexões necessárias entre conhecimento e intervenção centradas na realidade e repercussões no processo formativo. Interface-Comunicação, Saúde, Educação, v. 18, p. 177-186, 2014.

BRASIL. Fundação Nacional de Saúde. Diretrizes de educação em saúde visando à promoção da saúde: documento base - documento I/Fundação Nacional de Saúde -Brasília: Funasa, 2007.

. Ministério da Saúde (MS) Glossário temático: gestão do trabalho e da educação na saúde. Brasília DF, 2012. Disponível em: https://bvsms.saude.gov.br/bvs/publicacoes/glossario_gestao_trabalho_2ed.pdf Acesso em: 03/07/2021. . Lei; LEI, №. 5.692, DE 11 DE AGOSTO DE 1971. Fixa Diretrizes e Bases para o ensino de, v. 1, p. 6377, 1971. 
. Lei n. 9.394, de 20 de dezembro de 1996 (1996). Estabelece as diretrizes e bases da educação nacional. Brasília, DF: Ministério da Educação. Disponível em: http://portal.mec.gov.br/seed/arquivos/pdf/tvescola/leis/ lein9394.pdf Acesso em 20 de ago. 2021.

COSTA, Paula Valéria Dias Pena et al. A educação em saúde como ferramenta no combate ao câncer de mama: relato de experiência. Research, Society and Development, v. 9, n. 10, p. e6389108912-e6389108912, 2020.

DE SOUSA GONÇALVES, Romário et al. Educação em saúde como estratégia de prevenção e promoção da saúde de uma unidade básica de saúde. Brazilian Journal of Health Review, v. 3, n. 3, p. 5811-5817, 2020.

DIAS, Ernandes Gonçalves et al. Promoção de saúde na perspectiva da prevenção de doenças parasitárias por meio da educação em saúde com escolares do ensino fundamental. Revista de Epidemiologia e Controle de Infecção, v. 8, n. 3, p. 283285, 2018.

ENRIA, G.; STAFFOLANI, C. Contradicciones de los discursos que dificultan la transformación de las prácticas de promoción a la salud. Hacia la promoción de la salud, Calle, v. 15, n. 1, 2010.

FALKENBERG, Mirian Benites et al. Educação em saúde e educação na saúde: conceitos e implicações para a saúde coletiva. Ciência \& Saúde Coletiva, v. 19, p. 847-852, 2014.

FIGUEIREDO, Elisabeth Almeida; ANTUNES, Daniella Cavalcante; DE MIRANDA, Maria Geralda. Políticas públicas de educação em saúde para a prevenção de comorbidades e doenças cardiovasculares. Revista da Seção Judiciária do Rio de Janeiro, [S.I.], v. 23, n. 45, p. 141-160, jul. 2019. ISSN 2177-8337.

GOMES, Ana Cláudia Ferreira et al. Educação em saúde para prevenção do câncer de pênis: relato de experiência. Brazilian Journal of Health Review, v. 2, n. 4, p. 2961-2964, 2019. 
JANINI, Janaina Pinto; BESSLER, Danielle; VARGAS, Alessandra Barreto de. Educação em saúde e promoção da saúde: impacto na qualidade de vida do idoso. Saúde em Debate, v. 39, p. 480-490, 2015. LOPES, M. S. V.; SARAIVA, K. R. O.;

LIMA, D. C.; GARCIA, M. P. .; LIMA, E. S. de .; BEZERRA, C. C. . Health education as a tool for the prevention of cardiovascular diseases in the Elderly Health Care Program. Research, Society and Development, [S. I.], v. 9, n. 10, p. e079107382, 2020. DOI: 10.33448/rsd-v9i10.7382. Disponível em: https://rsdjournal.org/index.php/rsd/article/view/7382. Acesso em: 28 aug. 2021.

OBJETIVOS DA ONU - ODS. Objetivo 3. Assegurar uma vida saudável e promover o bem-estar para todas e todos, em todas as idades. Disponível em: <https://gtagenda2030.org.br/ods/ods3/>. Acesso em: 25 ago. 2021

PIMONT, Rosa Pavone. A educação em saúde: conceitos, definições e objetivos. Boletín de la Oficina Sanitaria Panamericana (OSP); 82 (1), ene. 1977, 1977.

RICCI, Ana Patrícia et al. Infecções sexualmente transmissíveis na gestação: educação em saúde como estratégia de prevenção na atenção básica. Brazilian Journal of Health Review, v. 2, n. 1, p. 565-570, 2019.

SALCI, Maria Aparecida; MEIRELLES, Betina Hörner Schlindwein; SILVA, Denise Maria Guerreiro Vieira da. Educação em saúde para prevenção das complicações crônicas do diabetes mellitus na atenção primáriaa. Escola Anna Nery, v. 22, 2018.

SCHALL, Virgínia T.; STRUCHINER, Miriam. Educação em saúde: novas perspectivas. 1999.

SOUSA, Marta Caires; GUIMARÃES, Ana Paula Miranda; AMANTES, Amanda. A saúde nos documentos curriculares oficiais para o ensino de ciências: da lei de diretrizes e bases da educação à base nacional comum curricular. Revista Brasileira de Pesquisa em Educação em Ciências, p. 129-153, 2019. 
SPERANDIO, Naiara; SPERANDIO, Natália Elvira. A educação em saúde enquanto prática transformadora da realidade social. Educação e Saúde: fundamentos e desafios, v. 1, n. 2, p. 7-14, 2018.

XIMENES, L. B. Análise do conceito de promoção da saúde. Texto Contexto Enferm., Florianópolis, v. 19, n. 3, jul./set. 2010, p. 461-468.

\section{ANNEXE - NOTE DE BAS DE PAGE DE RÉFÉRENCE}

7. Les gens qui faisaient partie de la doctrine de l'hygiénisme, une doctrine née au milieu du XIXe siècle, lorsque les dirigeants accordent une plus grande attention à la santé, ainsi qu'au moral de la population. A cette époque la maladie était considérée comme un phénomène social impliquant tous les aspects de la vie humaine, c'est à travers ce contexte que le traitement de l'eau, des eaux usées, de l'éclairage public se pose, afin de contrôler ou d'éliminer les pandemies (ABREU JUNIOR; CARVALHO, 2012).

Soumis: Septembre, 2021.

Approbation: Novembre 2021. 